\title{
Reconstruction of piecewise homogeneous images from partial knowledge of their Fourier Transform
}

\author{
Olivier Féron* and Ali Mohammad-Djafari* \\ * Laboratoire des Signaux et Systèmes, \\ Supélec, Plateau de Moulon, 91192 Gif-sur-Yvette, France
}

\begin{abstract}
Fourier synthesis (FS) inverse problem consists in reconstructing a multi-variable function from the measured data which correspond to partial and uncertain knowledge of its Fourier Transform (FT). By partial knowledge we mean either partial support and/or the knowledge of only the module and by uncertain we mean both uncertainty of the model and noisy data. This inverse problem arises in many applications such as : optical imaging, radio astronomy, magnetic resonance imaging (MRI) and diffraction scattering (ultrasounds or microwave imaging).

Most classical methods of inversion are based on interpolation of the data and fast inverse FT. But when the data do not fill uniformly the Fourier domain or when the phase of the signal is lacking as in optical interferometry, the results obtained by such methods are not satisfactory, because these inverse problems are ill-posed. The Bayesian estimation approach, via an appropriate modeling of the unknown functions gives the possibility of compensating the lack of information in the data, thus giving satisfactory results.

In this paper we study the case where the observations are a part of the FT modulus of objects which are composed of a few number of homogeneous materials. To model such objects we use a Hierarchical Hidden Markov Modeling (HMM) and propose a Bayesian inversion method using appropriate Markov Chain Monte Carlo (MCMC) algorithms.
\end{abstract}

Key words : Fourier Synthesis, Segmentation, Potts Markov Random Field, HMM, Gibbs sampling.

\section{INTRODUCTION}

In many applications of optical interferometry, X-ray crystallography ([5]), astronomy ([6]) or electron microscopy ([7]), one of the main mathematical part of the inversion problems, when linearized, become a Fourier synthesis one, where only partial information in image space and in Fourier domain are available. In astronomy and electron microscopy the problem of recovering a finite-support signal from its Fourier Transform magnitude arises. In optical astronomy, interferometric imaging techniques are employed. This yields to the magnitude of the Fourier Transform of the image with additional knowledge as non negative values and bounded spatial extent (finite-support). In many cases it can be shown that Fourier phase is significantly more relevant in representing a signal. Moreover in some situations it is possible to fully reconstruct a signal from its Fourier phase-only information. Specific conditions for unique representation and methods for signal reconstruction were introduced and tested in [4, 3, 8]. Most of these algorithms are iterative, alternating between the spatial and Fourier domains. One 
of the most successful algorithm is the Input-Output (I/O) algorithm of Fienup ([2]).

In this paper we study the case where only the magnitude, or a part of the magnitude, is observed, and we know the support of the 2D-object. The mathematical problem becomes the estimation of an image $f(\boldsymbol{r}), \boldsymbol{r} \in \mathcal{S} \subset \mathcal{R}^{2}$, from noisy and partial knowledge of its Fourier Transform magnitude $g(\omega), \omega \in \Omega \subset \mathcal{R}^{2}$ :

$$
\begin{aligned}
g(\omega) & =G(F(\omega))+\varepsilon(\omega)=[\mathcal{H} f](\omega)+\varepsilon(\omega) \\
F(\omega) & =\int f(\boldsymbol{r}) e^{-j \omega r} d \boldsymbol{r} \\
G(.) & =|.|
\end{aligned}
$$

Note that if we consider images, the pixels $r$ of the spatial domain belong to a finite lattice $S$ and we will note $S$ the number of pixels of this lattice. With the same idea we consider $\Omega$ as a discrete lattice, whose length is noted by $L$. In the following we use the vectorial notation :

$$
\boldsymbol{g}=\boldsymbol{H}(\boldsymbol{f})+\varepsilon,
$$

where $\boldsymbol{g}=\{g(\omega), \omega \in \Omega\}, \varepsilon=\{\varepsilon(\omega), \omega \in \Omega\}, \boldsymbol{f}=\{f(\boldsymbol{r}), \boldsymbol{r} \in \mathcal{S}\}$, and $\boldsymbol{H}: \mathcal{R}^{S} \longrightarrow \mathcal{R}_{+}^{L}$ represents the truncation of the magnitude of the Fourier Transform of the image $f$. In this paper we propose a Bayesian approach and compare the results with the Fienup algorithm.

\section{BAYESIAN APPROACH}

Within the observation model (2) and assuming $\varepsilon$ centered, white and Gaussian $p(\varepsilon)=$ $\mathcal{N}\left(0, \sigma_{\varepsilon}^{2} I\right)$, and $L$ the size of the observation $g$, we have :

$$
p(\boldsymbol{g} \mid \boldsymbol{f})=\mathcal{N}\left(\boldsymbol{H}(\boldsymbol{f}), \sigma_{\varepsilon}^{2} I\right)=\left(\frac{1}{2 \pi \sigma_{\varepsilon}^{2}}\right)^{\frac{L}{2}} \exp \left\{-\frac{1}{2 \sigma_{\varepsilon}^{2}}\|\boldsymbol{g}-\boldsymbol{H}(\boldsymbol{f})\|^{2}\right\}
$$

\section{Gaussian mixture model for homogeneous modeling}

Now we consider the case where we want a reconstructed image with statistically homogeneous regions. This can be modeled by introducing a new variable $\boldsymbol{z}=\{z(1), \cdots, z(S)\}, S$ being the number of pixels of $\boldsymbol{f}$, where each $z(r)$ takes a discrete value $k \in 1, \ldots, K$ corresponding to the index of the region. Then defining the following notations

$$
R_{k}=\{r: z(\boldsymbol{r})=k\}, \quad\left|R_{k}\right|=n_{k}, \text { and } \boldsymbol{f}_{k}=\{f(\boldsymbol{r}): z(\boldsymbol{r})=k\}
$$


and assuming that all the pixels with the same value $z(\boldsymbol{r})=k$ are inside a homogeneous region with mean value $m_{k}$ and variances $\sigma_{k}^{2}$, we can write

$$
\begin{aligned}
p\left(\boldsymbol{f}_{k}\right)=\mathcal{N}\left(m_{k} 1_{k}, \sigma_{k}^{2} \boldsymbol{I}_{k}\right) & =\left(\frac{1}{2 \pi \sigma_{k}^{2}}\right)^{\frac{n_{k}}{2}} \exp \left\{-\frac{1}{2 \sigma_{k}^{2}} \sum_{j}\left(f_{j}-m_{k}\right)^{2}\right\} \\
& =\left(\frac{1}{2 \pi \sigma_{k}^{2}}\right)^{\frac{n_{k}}{2}} \exp \left\{-\frac{1}{2 \sigma_{k}^{2}}\left\|\boldsymbol{f}_{k}-m_{k} 1_{k}\right\|^{2}\right\}
\end{aligned}
$$

and

$$
p(\boldsymbol{f})=\prod_{k} \mathcal{N}\left(m_{k} 1_{k}, \sigma_{k}^{2} \boldsymbol{I}_{k}\right)=\prod_{k}\left(\frac{1}{2 \pi \sigma_{k}^{2}}\right)^{\frac{n_{k}}{2}} \exp \left\{-\frac{1}{2 \sigma_{k}^{2}}\left\|\boldsymbol{f}_{k}-m_{k} 1_{k}\right\|^{2}\right\}
$$

where $1_{k}$ is a vector of $n_{k}$ elements all equal to one.

\section{Different modelings on the labels $z$}

To include more explicitly this classification, we write this dependence :

$$
p(f(\boldsymbol{r}) \mid z(\boldsymbol{r})=k)=\mathcal{N}\left(m_{k}, \sigma_{k}^{2}\right)
$$

We assume then that, given the labels, the pixels $f(\boldsymbol{r})$ are independent and we can go in different directions on the modeling of the labels $z$.

Independent case .

In this part we assume the labels independent, which means :

$$
P(z(\boldsymbol{r})=k)=p_{k}, \quad k=1, \ldots, K
$$

the prior distribution $\pi\left(p_{1}, \ldots, p_{K}\right)$ is chosen uniform. Then the a posteriori values of $p_{k}$ will be updated computing the number of pixels which belong in each class.

Potts Markov modeling case .

As we introduced the hidden variable $z$ for finding statistically homogeneous regions in images, it is natural to define a spatial dependency on these labels. The simplest model to account for this desired local spatial dependency is a Potts Markov Random Field model :

$$
P(\boldsymbol{z})=\frac{1}{T(\alpha)} \exp \left\{\alpha \sum_{\boldsymbol{r} \in \mathcal{S}} \sum_{\boldsymbol{s} \in \mathcal{V}(\boldsymbol{r})} \delta(z(\boldsymbol{r})-z(\boldsymbol{s}))\right\},
$$

where $\mathcal{S}$ is the set of pixels, $\delta(0)=1, \delta(t)=0$ if $t \neq 0, \mathcal{V}(\boldsymbol{r})$ denotes the neighborhood of the pixel $r$ (here we consider a neighborhood of 4 pixels), $T(\alpha)$ is the partition function or the normalization constant and $\alpha$ represents the degree of the spatial dependency of 
the variable $z$.

We have now all the necessary prior laws $p(\boldsymbol{g} \mid \boldsymbol{f}), p(\boldsymbol{f} \mid \boldsymbol{z})$, and $P(\boldsymbol{z})$ and to give an expression for $p(\boldsymbol{f}, \boldsymbol{z} \mid \boldsymbol{g})$. However these probability laws have in general unknown parameters such as $\sigma_{\varepsilon}^{2}$ in $p(\boldsymbol{g} \mid \boldsymbol{f})$ or $m_{k}$ and $\sigma^{2}{ }_{k}$ in $p(\boldsymbol{f} \mid \boldsymbol{z})$. We then have to assign prior laws to these "hyperparameters".

\section{Conjugate priors for the hyperparameters}

Let $\boldsymbol{m}=\left(m_{k}\right)_{k=1, \ldots, K}$ and $\sigma^{2}=\left(\sigma_{k}^{2}\right)_{k=1, \ldots, K}$ be the means and the variances of the pixels in different regions of the images $f$ as defined before. We define $\theta$ as the set of all the parameters which must be estimated :

$$
\theta=\left(\sigma_{\varepsilon}^{2}, \boldsymbol{m}, \sigma^{2}\right),
$$

The choice of prior laws for the hyperparameters is still an open problem. In [9] the authors used differential geometry tools to construct particular priors which contain as particular case the entropic and conjugate priors. In this paper we choose this last one. When applied the particular priors of ([9]) for our case, we find the following conjugate priors :

- Inverse Gamma IG $\left(\alpha_{0}^{\varepsilon}, \beta_{0}^{\varepsilon}\right)$ and $I \mathcal{G}\left(\alpha_{0}, \beta_{0}\right)$ respectively for the variances $\sigma_{\varepsilon}^{2}$ and $\sigma_{k}^{2}$

- Gaussian $\mathcal{N}\left(m_{0}, \sigma_{0}^{2}\right)$ for the means $m_{k}$.

\section{A POSTERIORI CONDITIONAL DISTRIBUTIONS}

As we want to use a Gibbs sampling ([1]) for estimating the set $(\boldsymbol{f}, \boldsymbol{z}, \boldsymbol{\theta})$ following the joint a posteriori distribution $p(\boldsymbol{f}, \boldsymbol{z}, \theta \mid \boldsymbol{g})$, we have to define the conditional $a$ posteriori distributions $p(\boldsymbol{f} \mid \boldsymbol{z}, \theta, \boldsymbol{g}), P(\boldsymbol{z} \mid \boldsymbol{f}, \theta, \boldsymbol{g})$ and $p(\theta \mid \boldsymbol{f}, \boldsymbol{z}, \boldsymbol{g})$, in order to sample these probabilities.

Sampling $z$ using $P(z \mid g, f, \theta)$ :

For this step we have :

$$
\begin{aligned}
P(\boldsymbol{z} \mid \boldsymbol{g}, \boldsymbol{f}, \theta) & \propto p(\boldsymbol{g} \mid \boldsymbol{f}, \boldsymbol{z}, \theta) P(\boldsymbol{z} \mid \boldsymbol{f}, \theta) \\
& =p(\boldsymbol{g} \mid \boldsymbol{f}) P(\boldsymbol{z} \mid \boldsymbol{f}, \boldsymbol{\theta}) \\
& \propto P(\boldsymbol{z} \mid \boldsymbol{f}, \theta)
\end{aligned}
$$

and

$$
P(\boldsymbol{z} \mid \boldsymbol{f}, \boldsymbol{\theta}) \propto P(\boldsymbol{z}) p(\boldsymbol{f} \mid \boldsymbol{z}, \boldsymbol{\theta})=P(\boldsymbol{z}) \prod_{\boldsymbol{r} \in \mathcal{S}} p(f(\boldsymbol{r}) \mid z(\boldsymbol{r}), \boldsymbol{\theta})
$$

We have two different priors on the labels, as defined before. For the independent case the probabilities $p_{k}$ are estimated computing the number $n_{k}$ of pixels which belong in each class $k$ and we have :

$$
p_{k}=\frac{n_{k}}{S}
$$


In the case of the Potts Markov Random Field (PMRF), we may note that an exact sampling of the a posteriori distribution $P(\boldsymbol{z} \mid \boldsymbol{f}, \theta)$ is impossible. However we may note that $P(\boldsymbol{z} \mid \boldsymbol{f}, \theta)$ is still a PMRF where the probabilities are weighted by $p(\boldsymbol{f} \mid \boldsymbol{z}, \boldsymbol{\theta})$. We use this fact to propose in the next section a parallel implementation of a Gibbs sampling for this PMRF.

sampling $\theta$ using $p(\theta \mid \boldsymbol{f}, \boldsymbol{g}, \boldsymbol{z})$ :

We have the following relation :

$$
p(\theta \mid \boldsymbol{f}, \boldsymbol{g}, \boldsymbol{z}) \propto p\left(\boldsymbol{m}, \boldsymbol{\sigma}^{2} \mid \boldsymbol{f}, \boldsymbol{z}\right) p\left(\sigma_{\varepsilon}^{2} \mid \boldsymbol{f}, \boldsymbol{g}\right)
$$

For the first term $p\left(\boldsymbol{m}, \sigma^{2} \mid \boldsymbol{f}, \boldsymbol{z}\right)$ we use the conditional distributions $p\left(\boldsymbol{m} \mid \boldsymbol{\sigma}^{2}, \boldsymbol{f}, \boldsymbol{z}\right)$ and $p\left(\sigma^{2} \mid \boldsymbol{m}, \boldsymbol{f}, \boldsymbol{z}\right)$. Using again the Bayes formula, the a posteriori distributions are calculated from the prior selection fixed before and we have

- $m_{k} \mid \boldsymbol{f}, \boldsymbol{z}, \sigma_{k}^{2}, m_{0}, \sigma_{0}^{2} \sim \mathcal{N}\left(\mu_{k}, v_{k}^{2}\right)$, with

$$
\mu_{k}=v_{k}^{2}\left(\frac{m_{0}}{\sigma_{0}^{2}}+\frac{1}{\sigma_{k}^{2}} \sum_{r \in R_{k}} f(r)\right), \quad v_{k}^{2}=\left(\frac{n_{k}}{\sigma^{2} k}+\frac{1}{\sigma^{2} 0}\right)^{-1}
$$

- $\sigma_{k}^{2} \mid \boldsymbol{f}, \boldsymbol{z}, m_{k}, \alpha_{0}, \beta_{0} \sim I \mathcal{G}\left(\alpha_{k}, \beta_{k}\right)$, with

$$
\alpha_{k}=\alpha_{0}+\frac{n_{k}}{2}, \quad \beta_{k}=\beta_{0}+\frac{1}{2} \sum_{r \in R_{k}}\left(f(\boldsymbol{r})-m_{k}\right)^{2}
$$

$\cdot \sigma_{\varepsilon}^{2} \mid \boldsymbol{f}, \boldsymbol{g} \sim I \mathcal{G}(\alpha, \beta)$, with

$$
\alpha=\frac{S}{2}+\alpha_{0}^{\varepsilon}, \quad \beta=\frac{1}{2}\|\boldsymbol{g}-\boldsymbol{f}\|^{2}+\beta_{0}^{\varepsilon}
$$

Sampling $\boldsymbol{f}$ using $p(\boldsymbol{f} \mid \boldsymbol{g}, \boldsymbol{z}, \boldsymbol{\theta})$ :

We can write the a posteriori law $p(f(\boldsymbol{r}) \mid g(\boldsymbol{r}), z(\boldsymbol{r}), \boldsymbol{\theta})$ as follows :

$$
\begin{aligned}
p(\boldsymbol{f} \mid \boldsymbol{g}, \boldsymbol{z}, \boldsymbol{\theta}) & \propto p(\boldsymbol{g} \mid \boldsymbol{f}, \boldsymbol{z}, \boldsymbol{\theta}) p(\boldsymbol{f} \mid \boldsymbol{z}, \boldsymbol{\theta}) \\
& \propto \exp \left\{-\frac{1}{2 \sigma_{\varepsilon}^{2}}\|\boldsymbol{g}-\boldsymbol{H}(\boldsymbol{f})\|^{2}-\sum_{k=1}^{K} \frac{1}{2 \sigma_{k}^{2}}\left\|\boldsymbol{f}_{k}-m_{k} 1_{k}\right\|^{2}\right\}
\end{aligned}
$$

If $\boldsymbol{H}(\boldsymbol{f})$ was a linear function of $\boldsymbol{f}$ then $p(\boldsymbol{f} \mid \boldsymbol{g}, \boldsymbol{z}, \boldsymbol{\theta})$ would be Gaussian and generating samples from it could be done easily. In our case $\boldsymbol{H}(\boldsymbol{f})$ is not linear and thus an exact sample of this distribution is impossible. A solution may be to study this distribution and then implement, at each step, a Hasting-Metropolis algorithm to have an exact sample. But this solution significantly increases the complexity of the algorithm. For this step we propose to approximate the algorithm computing the maximum of this a posteriori law :

$$
\hat{\boldsymbol{f}}=\arg \min _{f}\left\{\frac{1}{2 \sigma_{\varepsilon}^{2}}\|\boldsymbol{g}-\boldsymbol{H}(\boldsymbol{f})\|^{2}+\sum_{k=1}^{K} \frac{1}{2 \sigma_{k}^{2}}\left\|\boldsymbol{f}_{k}-m_{k} 1_{k}\right\|^{2}\right\}
$$

We can then implement an approximated Gibbs sampling for estimating the set of variables $(\boldsymbol{f}, \boldsymbol{z}, \boldsymbol{\theta})$. 


\section{APPROXIMATED GIBBS SAMPLING}

In the case of independent labels the sampling of $P(\boldsymbol{z} \mid \boldsymbol{f}, \theta)$ is easy. In the case of the Potts Markov Random Field, as we chose a first order neighborhood system on the labels, the a posteriori is still a PMRF with the same neighborhood. We can then decompose the whole set of pixels into two subsets forming a chess board ([1]). In this case if we fix the black (respectively white) labels, then the white (respectively black) labels become independent. This decomposition reduces the complexity of the Gibbs algorithm because we can simulate the whole set of labels in only two steps.

The Gibbs sampling, for both cases, is then the following : given an initial state $(\hat{\theta}, \hat{z})^{(0)}$,

\begin{tabular}{|c|c|}
\hline \multicolumn{2}{|r|}{ Approximate Gibbs sampling } \\
\hline repeat & until convergence \\
\hline 1. & compute $\hat{\boldsymbol{f}}_{i}^{(n)}=\arg \min _{f}\left\{\frac{1}{2 \sigma_{\varepsilon}^{2}}\|\boldsymbol{g}-\boldsymbol{H}(\boldsymbol{f})\|^{2}+\sum_{k=1}^{K} \frac{1}{2 \sigma_{k}^{2}}\left\|\boldsymbol{f}_{k}-m_{k} 1_{k}\right\|^{2}\right\}$ \\
\hline 2. & simulate $\boldsymbol{z}^{(n)} \sim p\left(\boldsymbol{z} \mid \hat{\boldsymbol{f}}^{(n)}, \hat{\boldsymbol{\theta}}^{(n-1)}\right)$ for the independent case \\
\hline or & simulate $\hat{\boldsymbol{B}}^{(n)} \sim p\left(\boldsymbol{z} \mid \hat{\boldsymbol{f}}^{(n-1)}, \hat{\boldsymbol{f}}^{(n)}, \hat{\boldsymbol{\theta}}^{(n)}\right)$ \\
\hline & simulate $\hat{x}^{(n)} \sim p\left(\boldsymbol{z} \mid \hat{\mathfrak{B}}^{(n)}, \hat{\boldsymbol{f}}^{(n)}, \hat{\boldsymbol{\theta}}^{(n)}\right)$ for the PMRF case \\
\hline 3. & simulate $\hat{\theta}^{(n)} \sim p\left(\theta \mid \hat{\boldsymbol{f}}^{(n)}, \mathfrak{z}^{(n)}, \boldsymbol{g}\right)$ \\
\hline
\end{tabular}

\section{SIMULATION RESULTS}

Here we present some results of our methods in the case of simulated data (figure (1-a)). We compare then the results of reconstruction with one of the most classical deterministic methods which if the I/O algorithm of Fienup ([2]). The main idea behind this algorihtm is using the principle of projections on the convex sets (POCS) which is described here in summary : Initialize the algorithm by $\boldsymbol{G}$ with the observed magnitude $M$ inside the Fourier support and zero outside, and the phase equals to zero. Repeat after convergence :

- 1. Inverse Fourier Transform $\boldsymbol{F}=I \mathcal{F}(\boldsymbol{G})$

- 2. $G=0$ outside the spatial support

- 3. Fourier Transform $\boldsymbol{G}=\mathcal{F}(\boldsymbol{F})$

- 4. $G=M$ inside the Fourier support

To compare the results with the original data we use the following correlation measure between two images $\boldsymbol{u}$ and $\boldsymbol{v}$ :

$$
d(\boldsymbol{u}, \boldsymbol{v})=\frac{\sum_{\boldsymbol{r} \in \mathcal{S}} \boldsymbol{u}(\boldsymbol{r}) \boldsymbol{v}(\boldsymbol{r})}{\|\boldsymbol{u}\|\|\boldsymbol{v}\|}
$$


Figure (2) shows the results of the different algorithms when the Signal-to-Noise Ratio vary. Figure (2-a) represents the case where we know the magnitude in the whole Fourier domain. In the figure (2-b) we consider the case where only a part of the magnitude $(40 \%)$ is observed. These results show that our algorithm gives better reconstructed images than the Fienup algorithm, particularly when the labels are modeled by the PMRF. Our algorithms are more robust to the noise and to the restriction of the observations in the Fourier domain.

Figure (1) shows the performances of the different algorithms in the case where only $40 \%$ of the magnitude is observed. Because we have introduced a label variable in our algorithms we can also obtain a classification result.

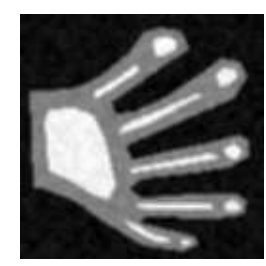

(a) Initial image

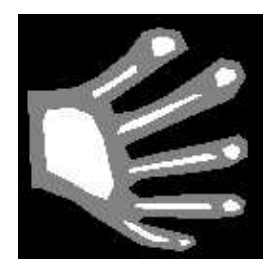

(b) initial segmentation

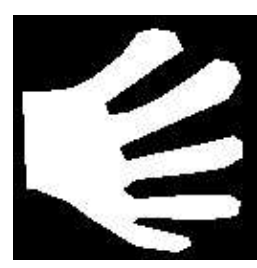

(c) Finite-support

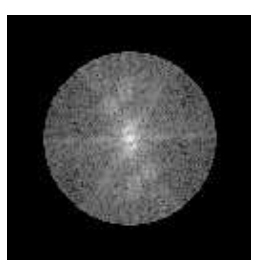

(d) Observed data
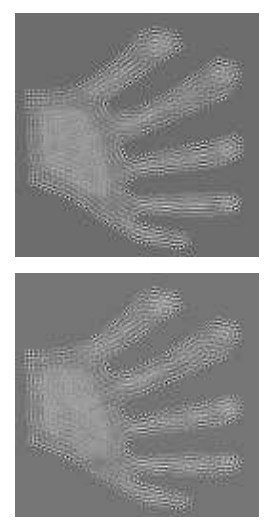

(e)
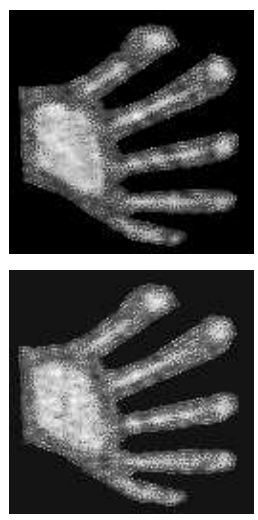

(f)
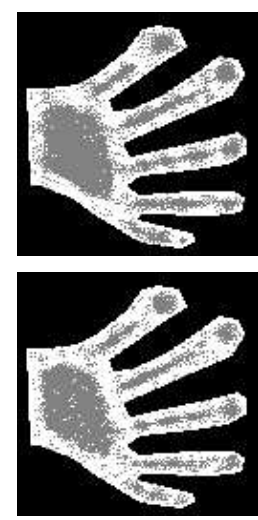

$(\mathrm{g})$
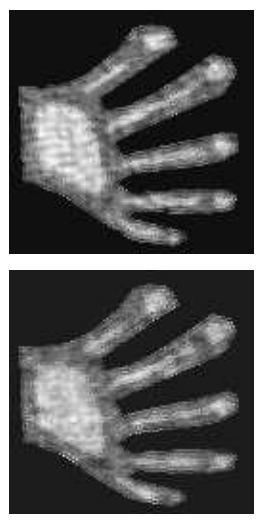

(h)
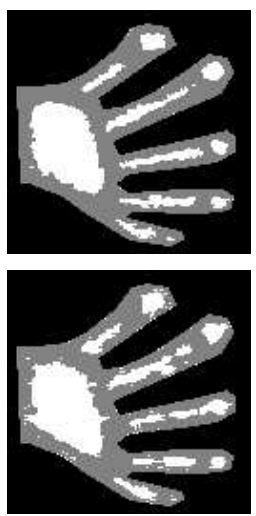

(i)

FIGURE 1. Results of Fourier Synthesis : (a) original image and (b) the perfect segmentation. (c) corresponding known spatial support. (d) Observed magnitude of the Fourier Transform. (e) result of the Fienup algorithm with $100 \%$ (up) and $40 \%$ (down) of the magnitude. (f) result of our method considering the labels independent and (g) the corresponding segmentation with $100 \%$ (up) and $40 \%$ (down) of the magnitude. (h) result of our method considering the PMRF on the labels (i) the corresponding segmentation with $100 \%$ (up) and $40 \%$ (down) of the magnitude.

When the magnitude is known in only a part of the Fourier domain the Fienup's algorithm becomes not efficient. In this case our methods give largely better results of reconstruction and a satisfactory segmentation. The modelization of the labels by a PMRF increases the performances of the method and we obtained more than 94\% of good classified labels in the case of high SNR data, versus $82 \%$ for the case of independent labels. 


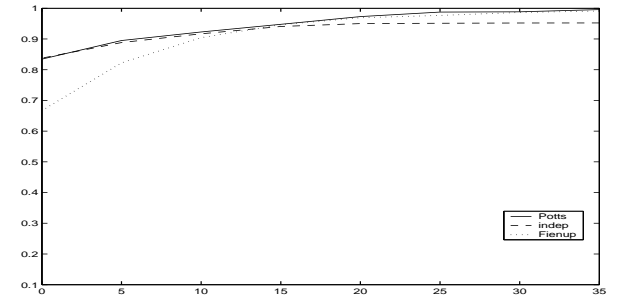

(a) Whole Fourier domain

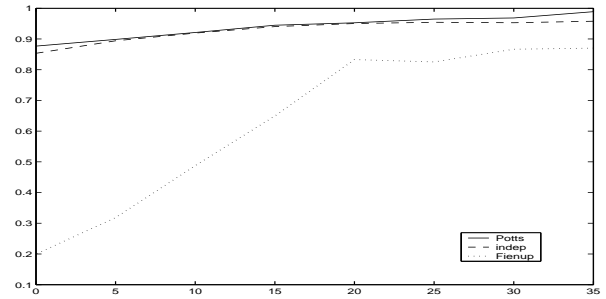

(b) $40 \%$ of the magnitude

FIGURE 2. Results of Fourier Synthesis : correlation measure of the three algorithms with (a) the whole Fourier domain and (b) only $40 \%$ of the magnitude

\section{CONCLUSION}

We proposed a Bayesian approach for Fourier Synthesis in the case where the observations are only a part of the magnitude of the Fourier Transform. We proposed an approximated Gibbs algorithm with a Hidden Markov Model which permits us to obtain a segmentation and we proposed two different modelings on the labels : independent and by a PMRF. We showed then that our method gives better results then the classical deterministic method of Fienup. We showed also how we can obtain an efficient segmentation in the case where only $40 \%$ of the magnitude is observed.

\section{REFERENCES}

1. Olivier Féron and Ali Mohammad-Djafari. Image fusion and unsupervised joint segmentation using HMM and MCMC algorithms. accepted in Journal of Electronic Imaging, 2004.

2. J.R. Fienup. Phase retrieval algorithms: A comparison. Appl. Opt., 21:2758-2769, August 1982.

3. M.H. Hayes. The reconstruction of multidimensional sequence from the phase or magnitude of its fourier transform. IEEE Transactions on Acoustics, Speech and Signal Processing, 30(2):140-152, April 1982.

4. M.H. Hayes, J.S. Lim, and A.V. Oppenheim. Signal reconstruction from the phase or magnitude transform. IEEE Transactions on Acoustics, Speech and Signal Processing, 30(2):140-152, April 1980.

5. J. Karle. Recovering phase information from intensity data. Science, 232:837-843, May 1986.

6. C.Y.C. Liu and A.W. Lohman. High resolution image information through the turbulent atmosphere. Opt.Comm., 8:372-377, August 1973.

7. D.L. Misell. A method for the solution of the phase problem in electron microscopy. J.Phys.D, 6:L6-L9, 1973.

8. T.F. Quatieri and A.V. Oppenheim. Iterative techniques for minimum phase signal reconstruction from phase or magnitude. IEEE Transactions on Acoustics, Speech and Signal Processing, 29:1187-1193, Dec 1981.

9. H. Snoussi and A. Mohammad-Djafari. Fast joint separation and segmentation of mixed images. Journal of Electronic Imaging, vol 13(2), pages 349-361, April 2004. 